\title{
Long timescale radio emission variability and spin-down changes in PSR J0738-4042
}

\author{
Aris Karastergiou ${ }^{1}$, Paul Brook ${ }^{1}$, Steve Roberts ${ }^{1}$, Sarah Buchner ${ }^{2}$ \\ and Simon Johnston ${ }^{1}$ \\ ${ }^{1}$ University of Oxford, United Kingdom \\ email: aris.karastergiou@gmail.com \\ ${ }^{2}$ HartRAO / University of Witwatersrand
}

\begin{abstract}
Recently, PSR J0738-4042 has grown a bright new emission component in its average pulse profile. Using data from Parkes and HartRAO, spanning back to the early 1980s, and applying statistical techniques to model the pulse profile shape with time, we have uncovered unexpected long-term variability, which is very well correlated with changes in the spin-down rate. We present these findings in the context of a growing population of radio-variable pulsars with correlated timing irregularities, including the intermittent pulsars, state-changing pulsars and other individual examples.
\end{abstract}

